\title{
Changing microbiological profile of pathogenic bacteria in diabetic foot infections: time for a rethink on which empirical therapy to choose?
}

\author{
P. Ramakant • A. K. Verma • R. Misra • K. N. Prasad • \\ G. Chand - A. Mishra - G. Agarwal - A. Agarwal • \\ S. K. Mishra
}

Received: 4 June 2010 / Accepted: 26 July 2010 / Published online: 11 September 2010

(C) Springer-Verlag 2010

\begin{abstract}
Aims/hypothesis We studied the bacterial aetiology and antibiotic sensitivity pattern of diabetic foot ulcers in India. Methods Records of 447 hospitalised patients between 1991 and 2008 were retrospectively analysed between two time periods (before and after 1999) to compare bacterial aetiology and antimicrobial sensitivity patterns. The first three consecutive cultures from the same wound during treatment were evaluated. Results Of 1,632 cultures, 66\% were polymicrobial, 23\% monomicrobial and $11 \%$ sterile. In the monomicrobial group, $14 \%(n=228)$ of cultures were Gram-negative, whereas $9 \%$ $(n=147)$ were Gram-positive. The most common pathogens in the first culture were Pseudomonas aeruginosa (20.1\%), Staphylococcus aureus (17.2\%) and Escherichia coli $(16.3 \%)$. Results for the third cultures showed persistence of P. aeruginosa (15.3\%) and E. coli (14.2\%). Gramnegative isolates dominated over Gram-positive ones ( $25.3 \%$ vs $15.1 \%, p<0.05)$. Antibiotic sensitivity patterns before and after 1999 were: piperacillin-tazobactam $74 \%$ vs $66 \%(p<0.005)$, imipenem $77 \%$ vs $85 \%$ (NS), cefoperazone-sulbactam $47 \%$ vs $44 \%(p<0.005)$, amikacin $62 \%$ vs $78 \%$ (NS), ceftriaxone $41 \%$ vs $36 \%(p<0.005)$, amoxicillinclavulanate $51 \%$ vs $43 \%(p<0.05)$ and clindamycin $43 \%$ vs $36 \%(p<0.005)$, respectively.
\end{abstract}

P. Ramakant · A. K. Verma $(\bowtie) \cdot$ G. Chand · A. Mishra •

G. Agarwal · A. Agarwal $\cdot$ S. K. Mishra

Department of Endocrine Surgery,

Sanjay Gandhi Post Graduate Institute of Medical Sciences, Raebarelly Road,

Lucknow 226014 Uttar Pradesh, India

e-mail: akverma@sgpgi.ac.in

R. Misra $\cdot$ K. N. Prasad

Department of Microbiology,

Sanjay Gandhi Post Graduate Institute of Medical Sciences,

Lucknow, India
Conclusions/interpretation Unlike in the West, in India Gram-negative bacteria were found to have always been dominant in the wounds of patients with diabetic foot infections. Infection with polymicrobial multidrugresistant Gram-negative bacilli is common. The policy of empirical antimicrobial therapy at tertiary care needs to be changed.

Keywords Bacterial aetiology - Diabetic foot infections . Empirical therapy $\cdot$ Multidrug-resistant organism
Abbreviations
DFI Diabetic foot infection
ESBL Extended spectrum $\beta$-lactamase
MDRO Multidrug-resistant organism
MRSA Methicillin-resistant $S$. aureus

\section{Introduction}

Foot infections in patients with diabetes are initially treated empirically and involve vital decisions regarding severity of infection, route of administration, co-morbidities and spectrum of organisms to be covered [1-4]. Therapy directed at known causative organisms can significantly improve the outcome and reduce infection-related morbidities. Reports from western countries have found that Staphylococcus aureus and $\beta$-haemolytic streptococci are the main causative pathogens [4-11]. In India, the choice of empirical antimicrobials is extrapolated from data available from western countries, which may or may not be appropriate for Indian patients [7-9, 12]. Since long-term studies from India on antimicrobial resistance and bacterial aetiology of diabetic foot infections (DFI) are scarce, we 
analysed existing hospital data on the microbiological profile of pathogenic bacteria isolated from wounds of patients with DFI over a period of time. Our study aims to identify the pathogenic bacteria and the changes in their antimicrobial sensitivity pattern in two time periods (up to year 1999 and thereafter).

\section{Methods}

Background From 1991 to 2008, a total of 447 DFI patients were hospitalised in the Endocrine Surgery ward of Sanjay Gandhi Post Graduate Institute of Medical Sciences, Lucknow, a tertiary care centre. Approval for this study was given by the Ethics Committee of the institute. Inclusion criteria included patients with DFI who were hospitalised for surgical management of foot ulcer. The complete data from 434 patients were available for inclusion in this retrospective study. All patients with incomplete data or who were not admitted for surgical intervention for treatment of foot ulcer were excluded.

Diabetic foot ulcers were graded using Wagner's classification prior to 1999 and by the University of Texas classification after 1999 [13, 14]. A questionnaire was designed, which included patient variables such as age, sex, duration of diabetes, glycaemic control, presence or absence of retinopathy, nephropathy (serum creatinine or presence of micro- or macro-albuminuria), neuropathy, peripheral vascular disease, cardiac abnormalities, gastropathy and duration of hospital stay.

A record was made of foot ulcer grade, duration of ulcer, infecting bacteria, and their culture and sensitivity profile including the antimicrobial agents used and duration of treatment. Data were then divided into two groups to observe changes in microbiological profile and sensitivity patterns over time. Group 1 included patients admitted between years 1991 and 1999; group 2 included patients admitted between 2000 and 2008 .

Method for procurement of culture specimens After admission to the ward, a minimum of three specimens were obtained from the wound for culture as follows: (1) at the time of admission; (2) at the time of first debridement; and (3) on subsequent debridement, i.e. weekly or as and when done. Specimens (pus, wound exudates or tissue biopsy) for microbiological studies were obtained from the ulcer region. To avoid isolation of colonising flora, the wound was first thoroughly cleaned with normal saline, after which samples were obtained from deeper pockets. Pus and exudates were collected from the margins and base of the ulcer, either in a syringe or using a sterile swab stick, which was then transported in a clean and sterile test-tube. Tissue biopsy/debrided fragments or a wedge of tissue was obtained during debridement using a sterile blade/knife from the base and/or margin of the ulcer, and transported in a sterile solution of normal saline. All specimens were transported without delay to the hospital's Microbiology Laboratory for further processing.

Culture and antibiotic sensitivity testing For aerobic culture, specimens were inoculated onto $5 \%$ (vol./vol.) sheep blood agar and MacConkey agar and incubated at $37^{\circ} \mathrm{C}$ for $48 \mathrm{~h}$. For anaerobic culture, specimens were inoculated on to Wilkin's Chalgreen agar (laced with kanamycin and vancomycin) and plates were incubated for 5 days in an anaerobic chamber at $37^{\circ} \mathrm{C}$.

Disc diffusion testing was carried out for all the isolates as currently recommended by the Clinical and Laboratory Standards Institute [15]. Gram-negative bacilli were tested for extended spectrum $\beta$-lactamase (ESBL) production and Staphylococcus species were tested for methicillin resistance by using $1 \mu \mathrm{g}$ oxacillin disc. Multidrug-resistant organisms (MOROs) were defined as methicillin-resistant $S$. aureus (MRSA), bacteria producing ESBL and methicillin-resistant coagulase-negative staphylococci. Appropriate quality controls were used where indicated.

Statistical analysis Data were analysed using SPSS software (version 16; SPSS, Chicago, IL, USA) and test of proportion was applied to calculate any significant differences $(p<0.05$ considered statistically significant).

\section{Results}

Data from 434 hospitalised patients with DFI were analysed. The demographic details and comorbid conditions are shown in Table 1. The details of ulcers with

Table 1 Clinical details of 434 patients infected with diabetic foot ulcers

\begin{tabular}{ll}
\hline Characteristic & Value \\
\hline Mean age (years) & $56 \pm 9.84$ \\
Mean foot ulcer duration (days) & $16 \pm 7.4$ \\
Men/women ratio & $4: 1$ \\
Retinopathy, $n(\%)$ & $334(77)$ \\
Cardiopathy, $n(\%)$ & $312(72)$ \\
Nephropathy, $n(\%)$ & $282(65)$ \\
Neuropathy, $n(\%)$ & $252(58)$ \\
Gastropathy, $n(\%)$ & $117(27)$ \\
Vasculopathy, $n(\%)^{\text {Poor glycaemic control, } n(\%)}{ }^{\text {a }}$ & $313(72)$ \\
\hline
\end{tabular}

Unless otherwise stated, values are mean $\pm \mathrm{SD}$

${ }^{\mathrm{a}} \mathrm{HbA}_{\mathrm{lc}} \geq 8.0 \%$ 
classification systems (Wagner's prior to 1999, University of Texas thereafter) are shown in Table 2. All patients underwent surgery in the form of debridement or minor/ major amputation. Other procedures included revision debridement and skin grafting.

The first three consecutive culture specimens from 434 patients yielded a total of 1,632 cultures, which were then analysed. The mean time interval $( \pm$ SEM) between first two cultures was 3.67 days $( \pm 0.04)$; between the second and third cultures the interval was 5.61 days $( \pm 0.06)$. Out of 1,632 cultures, $66 \%(n=1,093)$ were polymicrobial, whereas $23 \%$ were monomicrobial $(n=375)$. In the monomicrobial group, $14 \%(n=228)$ of cultures were Gram-negative; $9 \%(n=147)$ were Gram-positive. Cultures were sterile in $10.8 \%(n=179)$ specimens. First culture results proved that Gram-negative organisms dominated over Gram-positive ones $(25.3 \%$ vs $15.1 \%, p<0.05)$. The majority of the organisms were aerobes, but anaerobes were also isolated. Patient samples were sub-classified into two groups based on time periods of study (up to the year 1999 and thereafter). Table 3 describes the frequency of isolates obtained from cultures in both the above groups over two time periods (before and after 1999). Table 3 also shows

Table 2 Number of patients (\%) in the Wagner and UT classification systems

\begin{tabular}{ll}
\hline Classification system & Patients, $n(\%)$ \\
\hline Wagner classification grade (before 1999) & 164 \\
Grade 1 & $22(13.4)$ \\
Grade 2 & $46(28.0)$ \\
Grade 3 & $41(25)$ \\
Grade 4 & $35(21.3)$ \\
Grade 5 & $20(12.2)$ \\
University of Texas classification (after 1999) & 270 \\
Stage A & \\
Grade 1 & $8(2.9)$ \\
Grade 2 & $20(7.4)$ \\
Grade 3 & $22(8.1)$ \\
Stage B & \\
Grade 1 & $12(4.4)$ \\
Grade 2 & $26(9.6)$ \\
Grade 3 & $24(8.8)$ \\
Stage C & \\
Grade 1 & $14(5.2)$ \\
Grade 2 & $20(7.4)$ \\
Grade 3 & $28(10.4)$ \\
Stage D & \\
Grade 1 & $18(6.6)$ \\
Grade 2 & $30(11.1)$ \\
Grade 3 & $48(17.7)$ \\
\hline
\end{tabular}

that $P$. aeruginosa and $E$. coli persisted in all three cultures taken over a period of time during the entire treatment course, indicating a resistance pattern. Figure 1 (Gramnegative organisms) and Fig. 2 (Gram-positive organisms) indicate that resistance to antibiotics increased in isolates recovered from three consecutive cultures. Tests of significance when applied to these data are shown in separate Tables 4 and 5.

Commonly used empirical antimicrobial agents or their combinations included cloxacillin, metronidazole and amoxicillin-clavulanate in the first study period, whereas amoxicillin-clavulanate, clindamycin, pipearacillin-tazobactam or cefoperazone-sulbactam drugs or combinations were most commonly used in the second period. However, these drugs were suitably modified as indicated by subsequent culture and sensitivity reports.

The resistance profile of isolates obtained from first cultures in both time periods is shown in Fig. 3, which depicts a rising trend in the resistance pattern to commonly used antimicrobial agents in the long term. Multidrugresistant organisms in this study constituted up to $81 \%$, with $56 \%$ of $E$. coli being ESBLs and $25 \%$ of $S$. aureus being methicillin-resistant (MRSA).

\section{Discussion}

Severe and moderate DFIs are usually polymicrobial in nature, whereas mild DFI are mostly monomicrobial $[2,4$, 9-11]. In our study, $20.2 \%$ of the patients had mild infection. First culture results were monomicrobial in $78 \%$ of cases with mild DFI, while they were polymicrobial in $73 \%$ of cases with moderate to severe infections. Studies from western countries show that Gram-positive aerobes are the predominant organisms isolated from DFI [4-11]. In contrast, two recent Indian studies have shown a preponderance of Gram-negative aerobes. Gadepalli et al., in their study on 80 ulcer specimens, recovered 183 isolates, of which $28.7 \%$ were Gram-negative and only $13.8 \%$ Grampositive [16]. Shankar et al. also reported Gram-negative aerobes to be the most frequently isolated pathogens (51.4\%), followed by Gram-positive aerobes (33.3\%) and anaerobes [17]. Studies from Malaysia have also reported a predominance of Gram-negative bacteria $(52 \%)$ in patients with DFI, the most common pathogens isolated being Proteus spp., Klebsiella pneumoniae, E. coli and Enterobacter cloacae [18]. The difference observed in the prevalence of Gram-negative bacilli in DFI between diabetic patients from eastern and western countries remains largely unknown. However, environmental factors such as sanitary habits, e.g. use of water for peri-anal wash (ablution) after defaecation leading to contamination of hands with faecal flora, are proposed to be responsible for increased Gram- 
Table 3 Frequency of isolates in various cultures up to year 1999 and thereafter

\begin{tabular}{|c|c|c|c|c|c|c|}
\hline \multirow[t]{2}{*}{ Variable } & \multicolumn{4}{|c|}{ Isolates per culture } & \multicolumn{2}{|c|}{ Overall isolates } \\
\hline & 1st culture & 2nd culture & 3 rd culture & All cultures & Up to 1999 & After 1999 \\
\hline Cultures $(n)$ & 691 & 580 & 361 & 1632 & 689 & 943 \\
\hline Gram-negative & $415(25.3)$ & $332(20.3)$ & $186(11)$ & $932(57.1)$ & $349(50.6)$ & $603(64)$ \\
\hline P. aeruginosa & $135(20.1)$ & $89(15.3)$ & $53(15.3)$ & $277(16.9)$ & 123 & 147 \\
\hline E. coli & $116(16.3)$ & $97(16.7)$ & $50(14.2)$ & $263(16.1)$ & 70 & 153 \\
\hline Proteus spp. & $63(3.8)$ & $54(3.3)$ & $27(1.7)$ & $143(8.8)$ & 62 & 61 \\
\hline Klebsiella spp. & $48(2.9)$ & $40(2.5)$ & $21(1.3)$ & $109(6.7)$ & 20 & 89 \\
\hline Citrobacter spp. & $21(1.3)$ & $21(1.3)$ & $10(0.6)$ & $52(3.2)$ & 10 & 42 \\
\hline Acinetobacter spp. & $19(1.2)$ & $23(1.4)$ & $18(1.1)$ & $60(3.7)$ & 12 & 48 \\
\hline Enterobacter spp. & $13(0.8)$ & $8(0.5)$ & $7(0.4)$ & $28(1.7)$ & 52 & 63 \\
\hline Gram-positive & $247(15.1)$ & $182(11.2)$ & $82(5.0)$ & $511(31.3)$ & $280(40.6)$ & $286(30.3)$ \\
\hline S. aureus & $116(17.2)$ & 79 (13.6) & $30(1.8)$ & $225(13.8)$ & 101 & 144 \\
\hline Enterococcus spp. & $77(4.7)$ & $52(3.2)$ & $26(1.6)$ & $155(9.5)$ & 92 & 83 \\
\hline Coagulase-negative Staphylococcus & $30(1.8)$ & $33(2.0)$ & $19(1.2)$ & $82(5.0)$ & 45 & 37 \\
\hline Streptococcus spp. & $24(1.5)$ & $18(1.1)$ & $7(0.4)$ & $49(3.0)$ & 27 & 22 \\
\hline Anaerobes & $7(0.4)$ & $3(0.2)$ & $2(0.1)$ & $12(0.7)$ & 5 & 7 \\
\hline Sterile & $22(1.3)$ & $63(3.9)$ & $91(5.6)$ & $177(10.8)$ & 55 & 47 \\
\hline
\end{tabular}

Unless otherwise stated, values are mean. Values in parentheses are per cent (\%)

negative infections in the developing world compared with the West. Similar findings have been reported in continuous ambulatory peritoneal dialysis (CAPD) peritonitis by Prasad et al. [19].

Data from our study (Table 1) show that Gram-negative organisms were the major infective pathogens as compared with Gram-positive isolates $(51.7 \%$ vs $31.3 \%$; $p<0.01)$ in both time periods. Of all the aerobic bacteria recovered, the percentage of Gram-negative isolates increased from $50.6 \%$ before 1999 to $64 \%$ after $1999(p<0.01)$. The most common isolate in our study was P. aeruginosa $(16.9 \%)$ followed by E. coli (16.1\%) and Proteus spp. (8.8\%). Other Gramnegative aerobes recovered were Citrobacter spp., Enterobacter spp. and Acinetobacter spp.

The significance of separate analysis of cultures 1,2 and 3 in the same patient is that it enables the documentation of
Fig. 1 Overall antimicrobial sensitivity profile of Gram-negative bacterial cultures. Dark grey, culture 1; light grey, culture 2; medium grey, culture 3

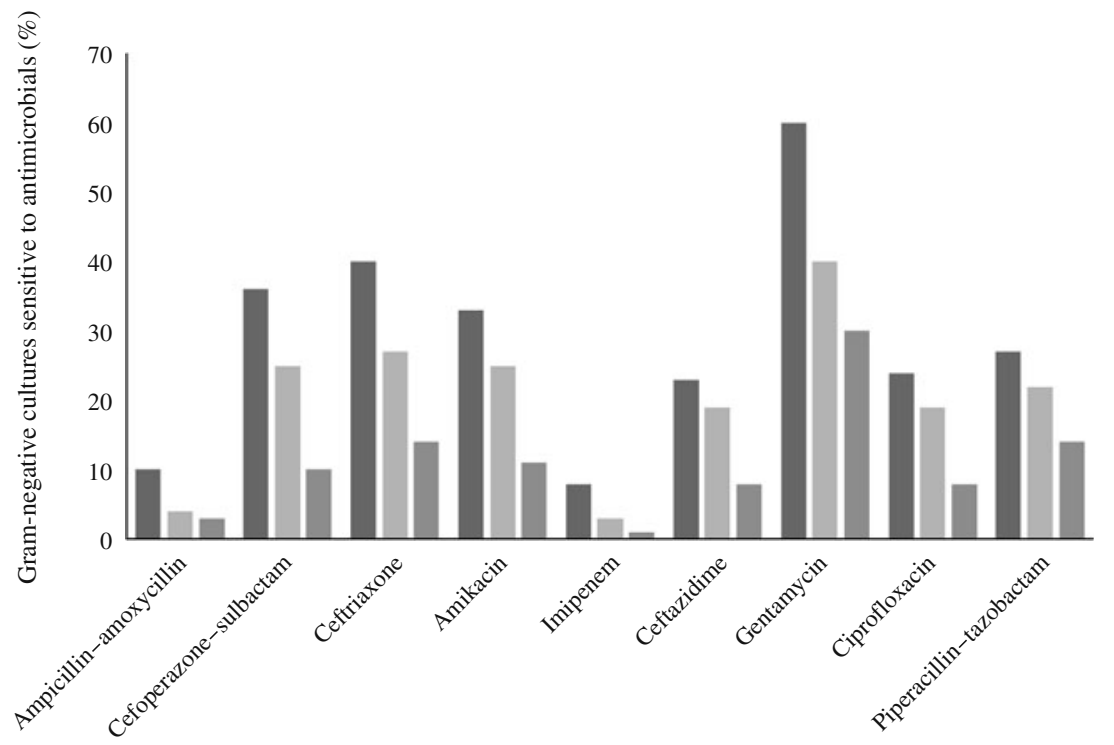

Antimicrobials 
Fig. 2 Antimicrobial sensitivity profile of Gram-positive bacteria. Dark grey, culture 1; light grey, culture 2 ; medium grey, culture 3

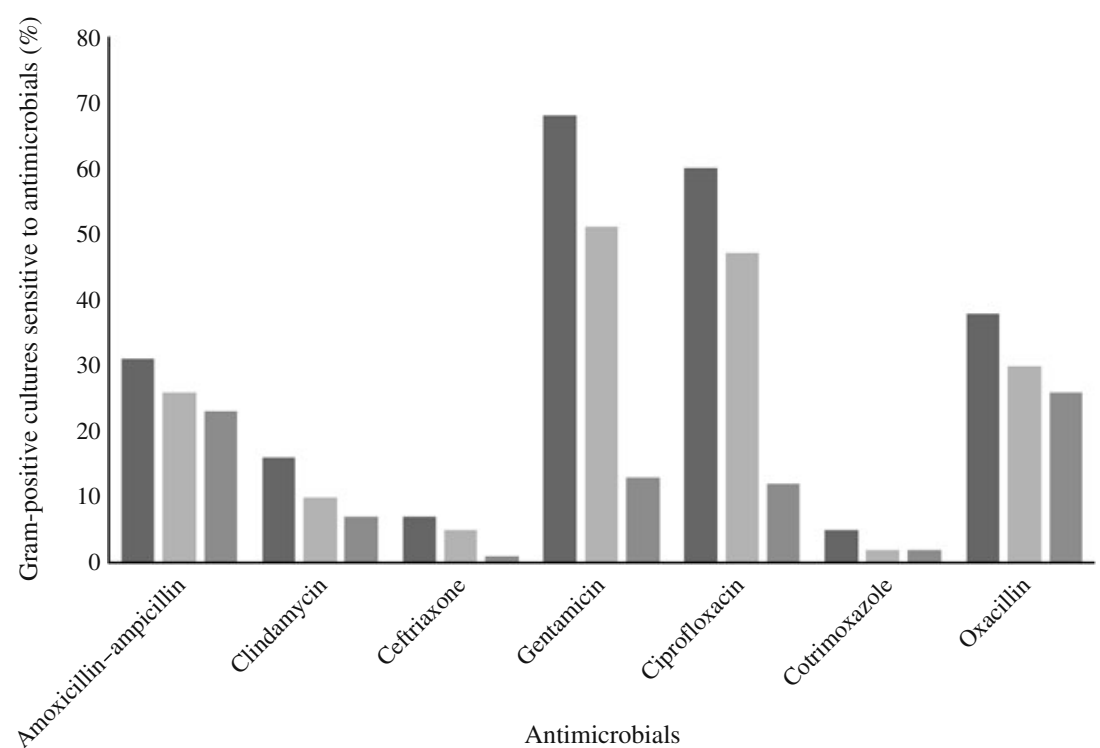

the emergence of resistance among Gram-negative and Gram-positive bacteria against commonly used antimicrobials (Figs 1 and 2, Tables 4 and 5). Follow-up cultures in the same patient at the time of admission, at debridement and at discharge also allowed us to monitor the change of microbial flora during treatment. Culture and sensitivity profile of isolates from second and third cultures showed persistence of $P$. aeruginosa and E. coli in up to $20 \%$ of isolates, which is worrisome as this indicates increasing resistance despite aggressive medical therapy. Shankar et al. also reported that $P$. aeruginosa was the most common bacterial isolate in their study [17]. Bansal et al., in their study on microbial flora in diabetic foot ulcers, reported that $76 \%$ of the isolates were Gram-negative, with $P$. aeruginosa being the most common pathogen [20].

Figure 3 shows a comparison of pre- and post-1999 populations that indicates increasing resistance to the more commonly used antimicrobial agents.
Recent studies from India have reported the prevalence of ESBL producers to be between $55 \%$ and $70 \%$ [21, 22]. MDROs in our study accounted for around $81 \%$, with $56 \%$ of E. coli isolates being ESBL producers. This is consistent with the report of Gadepalli et al. [16]. The persistence of $P$. aeruginosa and $E$. coli in third culture isolates confirms that multi-drug-resistant pathogens are extremely common in hospitalised patients with DFI, despite appropriate choice of antimicrobials. The high rates of antibiotic resistance observed in the present study may be due to the fact that ours is a tertiary care hospital as we see patients after the referring hospital has already tried and failed to control infection using a combination of different antimicrobials. Since facilities for microbiological studies at the first contact physician/surgeon are usually not available in district hospitals/smaller cities in India, indiscriminate antimicrobial therapy (i.e. without establishing the aetiology of the infection) eradicates susceptible Gram-positive cocci and, as a result, selects resistant
Table 4 Gram-negative bacteria: statistical analysis of sensitivity pattern

* Statistically significant $(p \leq 0.05)$

\begin{tabular}{|c|c|c|c|}
\hline \multirow[t]{2}{*}{ Antimicrobial agent } & \multicolumn{3}{|l|}{$p$ values per cultures } \\
\hline & Culture 1 vs culture 2 & Culture 2 vs culture 3 & Culture 1 vs culture 3 \\
\hline Ampicillin/amoxicillin & $0.001 *$ & 0.56 & $0.001 *$ \\
\hline Cefoperazone/sulbactam & 0.59 & $0.006 *$ & $0.003 *$ \\
\hline Ceftriaxone & $0.02 *$ & 0.98 & 0.09 \\
\hline Amikacin & $0.03 *$ & 0.09 & $0.03 *$ \\
\hline Imipenem & 0.1 & 0.34 & 0.59 \\
\hline Ceftazidime & $0.03 *$ & 0.09 & $0.03 *$ \\
\hline Gentamicin & $0.02 *$ & 0.98 & 0.09 \\
\hline Ciprofloxacin & 0.09 & $0.01 *$ & $0.01 *$ \\
\hline Piperacillin-tazobactam & 0.42 & 0.2 & $0.03 *$ \\
\hline
\end{tabular}


Table 5 Gram-positive bacteria: statistical analysis of sensitivity pattern

\begin{tabular}{|c|c|c|c|c|}
\hline & Amoxicillin-clavulanate & 0.18 & 0.24 & 0.8 \\
\hline & Clindamycin & 0.53 & 0.53 & 0.67 \\
\hline & Ceftriaxone & 0.36 & $0.04 *$ & 0.36 \\
\hline & Gentamicin & 0.19 & $0.01 *$ & $0.01 *$ \\
\hline & Ciprofloxacin & 0.35 & 0.64 & 0.21 \\
\hline & Cotrimoxazole & 0.16 & 0.61 & 0.64 \\
\hline$*$ Statistically significant & Oxacillin & 0.26 & 0.40 & 0.07 \\
\hline
\end{tabular}

Gram-positive cocci and Gram-negative bacilli, as well [23, 24]. The recent emergence of other mechanisms of drug resistance in Gram-negative bacilli such as metallo- $\beta$ lactamase (MBL) production and Amp $\mathrm{C}$ enzymes also needs to be explored [25]. All this is disconcerting because infection with these organisms limits the choice of antibiotic treatment and may lead to a worse outcome. These findings are important for patient management and underscore the need for institutional infection control committees to develop antibiotic treatment policies.

Several studies have also investigated the relationship between the specimen collection method and both numbers and types of organisms recovered from infected wounds. Some have found that tissue specimens are more sensitive and specific, containing fewer apparent contaminants and more pathogens than swab cultures $[26,27]$. Others have reported that with adequate preliminary debridement, the use of a wound swab is as reliable as the use of a tissue specimen $[27,28]$. In our study, swab specimens were collected only after thorough cleaning with sterile normal saline, after debridement of the wound and before application of an antiseptic agent. Culture material obtained from deeper tissues only was sent for microbiological study. However, sample collection procedures need to be carefully defined and observed, as skin contaminants may confuse the microbial profiles, possibly resulting in misinterpretation of culture reports with adverse effects on clinical decisions. Casual swab cultures should be strictly forbidden.

In conclusion, this study presents a comprehensive temporal microbiological survey of infected diabetic foot ulcers in hospitalised patients. Unlike in the West, in India we found that Gram-negative bacteria dominated in DFI patients. Hence all patients with DFI admitted to a tertiary care hospital in India may not require empirical therapy for Gram-positive coverage. A $\beta$-lactam agent with/without inhibitor combination or a quinolone as an empirical agent after establishing the patient's history of previous antibiotic usage would probably be more appropriate. However, treatment modes can be modified based on the severity of infection and on any available microbiological data such as recent culture results or current Gram-stained smear findings. Patients who are positive for MRSA, if the infection is community-acquired and sensitive to clindamycin, can be treated with this drug. In hospital-acquired infections with MRSA, glycopeptides are usually the drug of choice. In the event of Pseudomonas infection, an antipseudomonal drug can be added. In our study, piperacillintazobactam/cefoperazone-sulbactam adequately covered
Fig. 3 First culture isolates. Comparison of resistance pattern of pathogens isolated in two time periods, i.e. up to 1999 (dark grey) and after 1999 (light grey)

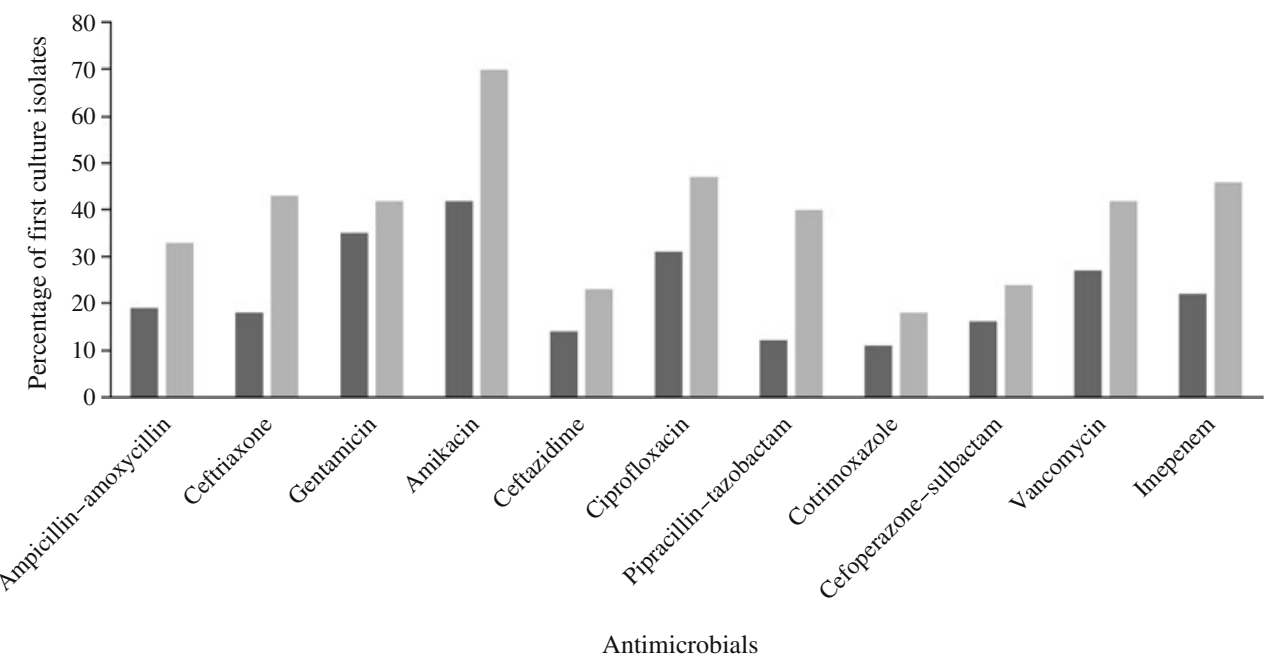


such infection. The results of this study therefore alert us to the need for proper management of antibiotics to optimise patient care and improve clinical outcome.

Acknowledgements The authors sincerely thank U. Singh (Department of Biostatistics) for assistance in data collection and analysis.

Duality of interest The authors declare that there is no duality of interest associated with this manuscript.

\section{References}

1. Lipsky BA (2007) Emperical therapy for diabetic foot infections: are there clinical clues to guide antibiotic selection? Clin Microbiol Infect 13:351-353

2. Lipsky BA, Anthony R, Berendt $\mathrm{H}$ et al (2004) Diagnosis and treatment of diabetic foot infections. Clin Infect Dis 39:885-891

3. Nicolau DP, Stein GE (2010) Therapeutic options for diabetic foot infections: a review with an emphasis on tissue penetration characteristics. J Am Podiatr Med Assoc 100:52-63

4. Dang CN, Prasad YD, Boulton AJ, Jude EB (2003) Methicillinresistant Staphylococcus aureus in the diabetic foot clinic: a worsening problem. Diabet Med 20:159-161

5. Citron DM, Goldstein EJC, Merriam CV, Lipsky BA, Abramson MA (2007) Bacteriology of moderate to severe diabetic foot infections and in vitro activity of antimicrobial agents. J Clin Microbiol 45:2819-2828

6. Lipsky BA, Pecoraro RE, Wheat LJ (1990) The diabetic foot: soft tissue and bone infection. Infect Dis Clin North Am 4:409-432

7. Grayson ML (1995) Diabetic foot infections: antimicrobial therapy. Infect Dis Clin North Am 9:143-161

8. Joseph WS, Axler DA (1990) Microbiology and antimicrobial therapy of diabetic foot infections. Clin Podiatr Med Surg 7:467-481

9. Goldstein EJ, Citron DM, Nesbit CA (1996) Diabetic foot infections: bacteriology and activity of 10 oral antimicrobial agents against bacteria isolated from consecutive cases. Diab Care 19:638-641

10. Sims D, Keating SE, DeVincentis AF (1984) Bacteriology of diabetic foot ulcers. J Foot Surg 23:149-151

11. Jones EW, Edwards R, Finch R, Jeffcoate WJ (1985) A microbiological study of diabetic foot lesions. Diabet Med 2:213-215

12. Nelson SB (2009) Management of diabetic foot infections in an era of increasing microbial resistance. Curr Infect Dis Rep $11: 375-382$

13. Smith RG (2003) Validation of Wagner's classification: a literature review. Ostomy Wound Manage 49:54-62
14. Oyibo SO, Jude EB, Tarawneh I, Nguyen HC, Harkless LB, Boulton AJ (2001) A comparison of two diabetic foot ulcer classification systems: the Wagner and the University of Texas wound classification systems. Diab Care 24:84-88

15. Clinical and Laboratory Standards Institute (2007) M100-S17. Performance standards for antimicrobial susceptibility testing. 16th informational supplement. Clinical and Laboratory Standards Institute, Wayne

16. Gadepalli R, Dhawan B, Sreenivas V, Kapil A, Ammini AC, Chaudhry R (2006) A clinico-microbiological study of diabetic foot ulcers in an Indian tertiary care hospital. Diab Care 29:1727-1732

17. Shankar EM, Mohan V, Premlatha G, Srinivasan RS, Usha AR (2006) Bacterial etiology of diabetic foot infections in South India. Eur J Intern Med 16:567-570

18. Raja NS (2007) Microbiology of diabetic foot infections in a teaching hospital in Malaysia: a retrospective study of 194 cases. J Microbiol Immunol Infect 40:39-44

19. Prasad KN, Prasad N, Gupta A, Sharma RK, Verma AK, Ayyagari A (2004) Fungal peritonitis in patients on continous ambulatory peritoneal dialysis: a single centre Indian experience. J Infect 48:96-101

20. Bansal E, Garg A, Bhatia S, Attri AK, Chander J (2008) Spectrum of microbial flora in diabetic foot ulcers. Indian J Pathol Microbiol $51: 123-126$

21. Kumar MS, Lakshmi V, Rajagopalan R (2006) Occurrence of extended spectrum beta lactamases among Enterobacteriaceae spp. isolated at a tertiary care institute. Indian J Med Microbiol 24:208-211

22. Mohanty S, Kapil A, Dhawan B, Das BK (2004) Bacteriological and antimicrobial susceptibility profile of soft tissue infections from Northern India. Indian J Med Sci 58:10-15

23. Patel V, Vaidya R, Naik D, Borker $P$ (2005) Irrational drug use in India: a prescription survey from Goa. J Postgrad Med 51:9-12

24. Sharma R, Sharma CL, Kapoor B (2005) Antibacterial resistance: current problems and possible solutions. Indian $\mathrm{J}$ Med Sci 59:120-129

25. Dallenne C, Costa AD, Decre D, Favier C, Guillaume A (2010) Development of a set of multiplex PCR assays for the detection of genes encoding important $\beta$-lactamases in Enterobacteriaceae. $\mathrm{J}$ Antimicrob Chemother 65:490-495

26. Senneville E, Melliez H, Beltrand E, Legout E, Valette M, Cazaubiel M (2006) Culture of percutaneous bone biopsy specimens for diagnosis of diabetic foot osteomyelitis: concordance with ulcer swab cultures. Clin Infect Dis 42:57-62

27. Pellizzer G, Strazzabosco M, Presi S et al (2001) Deep tissue biopsy vs superficial swab culture monitoring in the microbiological assessment of limb-threatening diabetic foot infection. Diabet Med 18:822-827

28. Slater RA, Lazarovitch T, Boldur I et al (2004) Swab cultures accurately identify bacterial pathogens in diabetic foot wounds not involving bone. Diabet Med 21:705-709 